\title{
Pflegepraxis durch Pflegewissenschaft in Zeiten von COVID-19 sichtbar machen
}

\author{
Manuela Hödl (D) - Silvia Bauer (D) - Doris Eglseer (D) - Martin Fangmeyer • Magdalena Flatscher-Thöni • \\ Jan Kellerer (D) - Christiane Kreyer (D) - Gerhard Müller (D) - Martin Pallauf $(\mathbb{D}$ - Matthias Rohringer (D) . \\ Ana Toromanova $\cdot$ Daniela Schoberer (iD
}

Eingegangen: 23. Oktober 2020 / Angenommen: 17. Mai 2021 / Online publiziert: 1. Juli 2021

(C) Springer-Verlag GmbH Austria, ein Teil von Springer Nature 2021

Zusammenfassung Die Pflegepraxis steht durch die COVID-19-Pandemie vor enormen Herausforderungen. Dennoch war die Pflegewissenschaft als Sprachrohr für die Praxis im öffentlichen Diskurs rund um die COVID-19-Pandemie kaum aktiv vertreten. Dementsprechend stellt sich die Frage, welche Projekte mit Fokus auf Pflege und COVID-19 an österreichischen Hochschulen durchgeführt wurden. Insgesamt wurden 6 Projekte mittels Schneeballverfahren identifiziert. Eine Studie zeigte, dass eine erhöhte Tragedauer von Masken mit einem erhöhten Stresslevel der Pflegepersonen zusammenhängt. Ein weiteres Studienergebnis zeigte, dass 50 \% der Laien, Masken fehlerhaft benutzten. Individuelle Erfahrungen, getroffene Maßnahmen und Lösungsansätze aus der Sicht der Pflegepersonen hinsichtlich ethischer Herausforderungen in Pflegeheimen wurden in einem weiteren Projekt erhoben. Durch diese und andere Projekte ergreift die Pflegewissenschaft mit ihren Erkenntnissen als Sprachrohr für Pflegekräfte die In-

\section{Hödl $(\bowtie) \cdot$ S. Bauer $\cdot$ D. Eglseer $\cdot$ D. Schoberer Institut für Pflegewissenschaft, Medizinische Universität Graz, Universitätsplatz 4, 8010 Graz, Österreich manuela.hoedl@medunigraz.at}

\section{Fangmeyer · A. Toromanova}

Department für Evidenzbasierte Medizin und Evaluation, Zentrum Cochrane Österreich, Donau-Universität Krems, Krems, Österreich

\section{Flatscher-Thöni}

Institut für Public Health, Medical Decision

Making und HTA, UMIT - Private Universität für

Gesundheitswissenschaften, Medizinische Informatik und Technik, Hall in Tirol, Österreich

J. Kellerer · C. Kreyer $\cdot$ G. Müller $\cdot$ M. Pallauf $\cdot$ M. Rohringer Institut für Pflegewissenschaft, UMIT - Private Universität für Gesundheitswissenschaften, Medizinische Informatik und Technik, Hall in Tirol, Österreich itiative und bringt sich in den COVID-19-Diskurs ein. Die Erkenntnisse aus den Projekten können zukünftig als Basis für politische Entscheidungen genutzt werden.

Schlüsselwörter COVID-19 · Pflege · Österreich

\section{Make nursing practice visible through nursing science in times of COVID-19}

Summary Due to coronavirus disease 2019 (COVID19) nursing practice is facing enormous challenges. Nevertheless, nursing science was hardly actively represented as a mouthpiece for the practice in the public discussions on the COVID-19 pandemic. Therefore, the question arises which projects with a focus on nursing and COVID-19 have been carried out at Austrian universities. Overall, six projects were identified using the snowball method. Of the studies one showed that an increased wearing time of masks is associated with an increased stress level for nursing personnel. An additional study result showed that almost $50 \%$ of lay people used masks incorrectly. Individual experiences, interventions undertaken and solution approaches from the perspective of the nursing staff with respect to ethical challenges in nursing homes were collected in a further project. This and other projects show that nursing science, with its knowledge and as a voice for nursing practice, has taken the initiative and contributes to the COVID-19 discourse. Additionally, the findings of the projects can be used as a basis for making future political decisions.

Keywords COVID-19 · Nursing care $\cdot$ Austria 
Beschäftigte als auch Entscheidungsträger*innen im Gesundheitssystem tragen die Verantwortung für eine qualitativ hochwertige Behandlung, Pflege sowie Betreuung und somit für die Sicherheit von Menschen mit Pflegebedarf [1]. Dies wurde insbesondere während der COVID-19-Pandemie sichtbar, als die weltweite Kampagne „clap for medical staff“ gestartet wur-

de, die im Angesicht solch herausfordernder Zeiten durchwegs kritisch zu betrachten ist [2]. Kurz darauf wurde der Slogan in verschiedensten Ländern in „clap for carers or health care workers“ umbenannt. Dies ist von besonderer Bedeutung, da Pflegende und Hebammen gemeinsam die größte Gruppe an Gesundheitspersonal darstellen [3, 4]. Diese stehen durch die aktuelle COVID-19-Pandemie bei der Betreuung von pflegebedürftigen Personen vor zusätzlichen Herausforderungen [5].

Pflegepersonen sowie auch viele Menschen in der Allgemeinbevölkerung haben Angst, an COVID-19 zu erkranken/zu versterben oder im Falle einer Erkrankung von Bezugspersonen getrennt $\mathrm{zu}$ werden $[5,6]$. Zusätzlich dazu sind Pflegepersonen zusätzlich mit weiteren Belastungen konfrontiert wie psychischer Stress, dem insbesondere Pflegepersonen, die direkt am Pflegebett mit COVID-19-Patient*innen arbeiten, ausgesetzt sind [5, 7].

Bei Pflegepersonen kommen noch weitere Faktoren hinzu, die Stress auslösen können. Dazu gehören das dauerhafte Tragen von persönlicher Schutzausrüstung (PSA), längere Arbeitszeiten, reduzierte Selbstfürsorge infolge von Zeit- und Energiemangel, die Angst, die eigenen An-/Zugehörigen mit COVID-19 anzustecken, oder auch unzureichende Informationen über längerfristige Folgen einer COVID-19-Erkrankung [6]. Dieser psychische Stress kann bei Pflegepersonen unter anderem Krankheiten wie Depressionen, Angststörungen oder Schlafstörungen auslösen und für die Betroffenen eine enorme Belastung darstellen [7, 8].

Ein weiterer belastender Faktor für Pflegepersonen und andere Akteur*innen im Gesundheitssystem war und ist das Fehlen von wissenschaftlichen Erkenntnissen und evidenzbasierten Empfehlungen zu präventiven und therapeutischen Maßnahmen im Zusammenhang mit der COVID-19-Pandemie [9]. Auf der anderen Seite wurde durch die COVID-19-Pandemie sichtbar, dass politische Entscheidungen auf wissenschaftlichen Erkenntnissen getroffen werden sollen [10]. Andererseits, hat sich die Pflegewissenschaft in Österreich bis dato kaum in den COVID-19Diskurs eingebracht [10]. In der österreichischen Presseagentur waren lediglich 4 von 162 Pressemeldungen vom österreichischen Gesundheits- und Krankenpflegeverband. Damit wird deutlich, dass die Pflegepraxis und die Pflegewissenschaft kaum die Initiative ergriffen haben, um sich medial sichtbar zu präsentieren [11]. Zusätzlich sind sowohl die Pflegepraxis als auch die Pflegewissenschaft nicht in der eigens eingerich- teten Coronavirus-Taskforce des Bundesministeriums vertreten [12].

Vor diesem Hintergrund ist es in dieser speziellen und herausfordernden Situation der COVID-19Pandemie notwendig, nationale pflegewissenschaftliche Forschungsarbeiten sichtbar zu machen, um dadurch einen besseren und objektiven Einblick in die Pflegepraxis zu bekommen. Dieser Artikel zielt darauf $\mathrm{ab}$, einen Überblick über pflegewissenschaftliche Projekte und einzelne Studien während der COVID19-Pandemie an österreichischen Hochschulen zu geben. Damit stellt sich folgende Frage „Welche pflegewissenschaftlichen Projekte wurden an österreichischen Hochschulen während der COVID-19-Pandemie durchgeführt?“

\section{Methode}

Die Autorinnen (MH, SB, DS) und weitere Kolleginnen vom Institut für Pflegewissenschaft der Medizinischen Universität Graz kontaktierten Anfang Juli 2020 verschiedene Kolleg*innen an österreichischen Hochschulen per E-Mail. In dieser E-Mail wurde das Ziel des Artikels, nämlich Forschungsarbeiten mit einem klaren Bezug zu Pflege und COVID-19 zu beschreiben, erläutert. Zusätzlich wurde eine Auflistung mitgesendet, in der die zu beschreibenden Aspekte des jeweiligen Projekts geklärt wurden: (1) Titel des Projekts, (2) Ziel des Projekts, (3) Methode, (4) falls bereits vorhanden, Ergebnisse und (5) Angaben zu einer Kontaktperson inklusive Link zum Projekt. Die kontaktierten Personen wurden ebenso aufgefordert, die E-Mail an Kolleg*innen weiterzuleiten (Schneeballverfahren).

\section{Ergebnisse}

Im nachfolgenden Abschnitt werden einzelne Projekte, deren Methode sowie die Ergebnisse, sofern bereits vorhanden, beschrieben.

\section{Projekt 1}

Das übergeordnete Ziel dieser Querschnittstudie war es, die „PflegEqualität während der COvid-19 Krise, unter besonderer Berücksichtigung von psychischem Stress der Pflegepersonen“ (PECOS), darzustellen.

Die Datenerhebung erfolgte vom 12.05.2020 bis 13.07.Juli 2020. Dabei füllten Pflegepersonen aus verschiedenen Gesundheitseinrichtungen (z. B. Krankenhaus, Pflegeheim, Hauskrankenpflege), welche direkt mit Patient*innen oder Bewohner*innen arbeiten, einen Online-Fragebogen aus.

Es wurden Alter, Geschlecht, aber auch allgemeine Aspekte wie das Beschäftigungsausmaß, die berufliche Qualifikation (Dipl. Pflegeperson, Pflegefachassistenz etc.) und die Einrichtungsart (Krankenhaus, Pflegeheim), in der die Person beschäftigt ist, erhoben. Der Fragebogen basiert auf dem Modell von Donabedian und inkludiert Struktur-, Prozess und 
Ergebnisindikatoren [13]. Zu den Strukturindikatoren zählen u.a. Fragen, ob es in der jeweiligen Gesundheitseinrichtung Leitlinien zum Umgang mit COVID19-Verdachtsfällen und Schulungen zu Schutzmaßnahmen gab. Prozessindikatoren beinhalten Fragen zu Begleitmaßnahmen, die von Pflegepersonen umgesetzt werden (z. B. transparente Informationsweitergabe), persönlichen Schutzmaßnahmen (z.B. Tragen von Mund-Nasen-Schutz [MNS]) und allgemeinen Schutzmaßnahmen (z. B. Händehygiene nach Kontakt mit Patient*innen). Die Struktur- und Prozessindikatoren wurden, basierend auf Empfehlungen der WHO [14, 15], dem Bundesministerium für Soziales, Gesundheit, Pflege und Konsumentenschutz [16-19] und anderen internationalen Publikationen $[8,20]$ erstellt. Der wahrgenommene Stress als Ergebnisindikator wurde mithilfe der Perceived Stress Scale (PSS) erhoben. Die PSS umfasst 10 Fragen und steht in deutscher Sprache zur Verfügung [21]. Zusätzlich wurde die PSS bereits bei Pflegepersonen verwendet und ist umfassend wissenschaftlich überprüft [22-24]. Ein positives Votum von der zuständigen Ethikkommission liegt vor.

IBM SPSS Statistik [25] wurde zur statistischen Analyse verwendet. Deskriptive Analysen aller Variablen wurden durchgeführt. Kategorielle Variablen werden als Prozentwerte dargestellt. Numerische Variablen werden je nach Verteilung als Mittelwert und Standardabweichung oder als Median und Interquartilsabstand angeführt.

Insgesamt nahmen 2605 Pflegepersonen aus verschiedenen österreichischen Gesundheitseinrichtungen an der Umfrage teil. Knapp zwei Drittel der Personen gaben an, dass eine ausreichende Anzahl an Mund-Nasen-Schutz (MNS) vorhanden war. Ebenso knapp zwei Drittel der Personen litten unter mäßigem bis hohem Stress. Zusätzlich konnte unter anderem beschrieben werden, dass eine längere Tragedauer der Masken und eine Erhöhung des Arbeitsausmaßes aufgrund von COVID-19 signifikant mit einem höheren Stresslevel der Pflegepersonen in Zusammenhang stand. $\mathrm{Zu}$ diesem Projekt gibt es bereits mehrere Publikationen bzw. Einreichungen [26-28]. Eine weitere Analyse dieses Projekts zeigte, dass bei $31,3 \%$ und $21,7 \%$ der Pflegepersonen Informationen nicht zeitgerecht und Maßnahmen nicht transparent kommuniziert wurden [29]. Dies kann wiederum den Stresslevel erhöhen. Zusätzliche Pausen und Erholungsphasen zur Stresslinderung gaben $55 \%$ der Pflegepersonen an [29]. Wohingegen psychosoziale Unterstützung oder Sonderurlaube von $34,9 \%$ sowie 33,7 \% der Pflegepersonen als Möglichkeit zum Schutz vor psychischer und physischer Überlastung aufgrund von COVID-19 angegeben wurden [29].

\section{Projekt 2}

Im ROSEN-Projekt ging es darum, die ARbeitsanfOrderungen von PflegeperSonen in PflEgeheimen während der COVID-19-PaNdemie zu untersuchen. Dieses Projekt war eine qualitative Studie mit 18 Pflegepersonen aus Pflegeheimen in der Steiermark und in Kärnten. Ein positives Votum von der zuständigen Ethikkommission liegt vor.

Einschlusskriterien für das Pflegeheimpersonal waren: Einwilligungsfähigkeit, Fähigkeit, Deutsch zu verstehen und $\mathrm{zu}$ sprechen, und die Qualifikation als „Diplomierte Gesundheits- und Krankenpflegeperson“ oder „Pflegefachassistenz/Pflegehilfe“ oder „Heimhilfe“ sowie direkte pflegerische Versorgung von Bewohner*innen während der COVID-19-Pandemie. Pflegeheimpersonal, welches im Management tätig war, wurde ausgeschlossen.

Um einen möglichst breiten Einblick in die pflegerische Versorgung in Pflegeheimen während der 1. COVID-19-Welle zu erhalten, wurde eine repräsentative Stichprobe mittels eines theoretisch zweckgerichteten Stichprobenplans gezogen. Dieser basierte auf Informationen des Bundesministeriums für Arbeit, Soziales, Gesundheit und Konsumentenschutz [30] sowie von Statistik Austria [31]. Die 4 Hauptvariablen zur Erstellung des Stichprobenplans waren Qualifikation, Geschlecht, Alter und die Lokalisation des Pflegeheims (Stadt vs. Land).

Neben den demografischen Angaben werden zusätzlich in einem kurzen Fragebogen die Qualifikation, die Berufserfahrung sowie der Kontakt mit COVID-19-(Verdachts-)Fällen erhoben. Die Arbeitsanforderungen wurden mit einem semistrukturierten Leitfaden $\mathrm{zu}$ Belastungen von Pflegepersonen erhoben [32]. Die Daten werden inhaltsanalytisch, basierend auf Mayring und Schreier [33, 34], analysiert.

Die Interviews wurden im Sommer 2020 durchgeführt und dauerten zwischen 22 und $68 \mathrm{~min}$. Mehr als drei Viertel der interviewten Personen waren weiblich $(77,8 \%)$ und im Durchschnitt 41 Jahre. Je $44 \%$ waren DGKP sowie Pflege(fach)assistent*innen.

Vorläufige Ergebnisse lassen darauf schließen, dass der Umgang mit An-/Zugehörigen, aber auch Maßnahmen zu setzen, um die Belastungen der Bewohner*innen so gering wie möglich zu halten, belastende Arbeitsanforderungen für das Pflegeheimpersonals darstellten. Detaillierte Ergebnisse sind im Sommer $2021 \mathrm{zu}$ erwarten.

\section{Projekt 3}

Im Rahmen eines Moduls wurde mit Studierenden der Pflegewissenschaft eine qualitative deskriptive Studie entwickelt, geplant und in der Steiermark und in Kärnten durchgeführt, welche sich mit den Auswirkungen von COVID-19 auf diplomierte Gesundheitsund Krankenpflegepersonen (DGKP) im Krankenhausbereich beschäftigte.

Einschlusskriterien für die DGKP waren: Einwilligungsfähigkeit, Fähigkeit, Deutsch zu verstehen und zu sprechen, und Kontakt mit Verdachtsfällen oder tatsächlich positiv getesteten COVID-19- 
Patient*innen im Rahmen ihrer Berufsausübung gehabt zu haben. Es wurde ein Stichprobenplan [35] erstellt, um eine möglichst heterogene Gruppe, hinsichtlich Alter, Geschlecht, private und öffentliche Krankenhäuser, städtische und ländliche Regionen zu erhalten.

Vor dem Interview wurde die informierte Zustimmung der DGKP eingeholt und anschließend ein Fragebogen zu den persönlichen Merkmalen wie Geburtsjahr, Geschlecht, Berufserfahrung oder aber auch der Kontakthäufigkeit mit COVID-19-Patient*innen im Berufsalltag ausgefüllt. Die Einzelinterviews, basierend auf einem standardisierten Interviewleitfaden, wurden durch geschulte Studierende durchgeführt. Der Interviewleitfaden bezog sich auf Auswirkungen von COVID-19 auf das Befinden und Verhalten, die mentale Gesundheit als auch auf körperliche und soziale Folgen [32]. Die qualitative Inhaltsanalyse nach Mayring [36] wurde zur Analyse der Interviews genutzt.

Es nahmen 18 DGKP zwischen 18 und 53 Jahren an dieser Studie teil, wovon der Großteil weiblich war. Es konnte gezeigt werden, dass es infolge des Arbeitens auf COVID-Stationen zu neuen Aufgaben und geänderten Verantwortungen insbesondere in den Bereichen der multiprofessionellen Zusammenarbeit und Patient*innenedukation kam. Neben der Angst, andere zu infizieren, oder der sozialen Isolation im privaten Umfeld wurden Kopfschmerzen, erhöhtes Schwitzen und Hautveränderungen in Zusammenhang mit dem Tragen der Schutzkleidung als Auswirkungen genannt. Die Pflegepersonen gaben auch an, dass ausreichend Information, regelmäßige Teambesprechungen und positive Bestärkung vonseiten des Unternehmens als unterstützend empfunden wurden und Sicherheit vermittelten.

Basierend auf den Projekten 1 bis 3, wurden bereits ein Interview [37] als auch ein Artikel in einer Tageszeitung [38] veröffentlicht.

\section{Projekt 4}

Eine Studie des Evidenzbasiertes Informationszentrum für Pflegende an der Donau-Universität Krems zielte darauf $a b$, eine rasche Evidenzsynthese zu der Fragestellung „Kann durch eine antiseptische Mundspülung mit Chlorhexidin oder Octenidindihydrochlorid bei Patient*innen mit COVID-19 eine Infektionsübertragung auf das Gesundheitspersonal verhindert werden?" zu erstellen. Ausgehend von einer Anfrage einer praktisch tätigen Pflegeperson wurde eine systematische Recherche inklusive COVID19-spezifischer Datenbanken [39-42] durchgeführt. Die Evidenzsynthese zeigte, dass keine Evidenz zu dieser Fragestellung vorliegt. Jedoch konnte eine laufende Untersuchung identifiziert werden. Aufgrund der unsicheren Ergebnisse, der Bedeutsamkeit des Themas und der rasch voranschreitenden Forschung [43] wurde diese zuerst einmalige Evidenzsynthese in ein Living Rapid Review umgewandelt, um neue Forschungsergebnisse möglichst rasch und regelmäßig $\mathrm{zu}$ integrieren. Aktuell erfolgen regelmäßig in kurzen Abständen Recherchen in COVID-19-spezifischen und halbjährlich umfangreiche systematische Literatursuchen in verschiedenen Datenbanken. Spätestens im August 2021 erfolgt ein Update. Derzeit ist eine randomisierte kontrollierte Studie mit hohem BiasRisiko zu der Fragestellung publiziert, und 6 aktuell laufende Studien sind registriert. Die derzeit vorliegenden Ergebnisse zeigen, dass Gurgeln mit $15 \mathrm{ml}$ Chlorhexidin innerhalb von $6 \mathrm{~h}$ zu keiner Reduktion der Viruskonzentration im Speichel von Patient*innen mit COVID-19 führt [44]. Demnach ist es aktuell nach wie vor unklar, ob antiseptische Mundspülungen eine COVID-19-Infektionsübertragung auf das Gesundheitspersonal verhindern.

\section{Projekt 5}

Im Rahmen eines Studierendenprojekts wurde das Verhalten von Laien im Umgang mit Mund-NasenSchutz-Masken (MNS) im öffentlichen Raum untersucht [45]. In einer standardisierten, nicht-teilnehmenden Beobachtungsstudie wurde das Verhalten von Personen bei der Verwendung von MNS vor Lebensmittelgeschäften in Tirol und Vorarlberg beobachtet.

Insgesamt wurden 2080 Personen an 24 verschiedenen Beobachtungsorten über einen Zeitraum von 6 Wochen beobachtet. Bei fast einem Drittel aller Beobachtungsobjekte ( $n=648 ; 31,2 \%)$ wurde ein Fehlverhalten vor der Positionierung des MNS (Beobachtungssequenz [BS] 1: Transport) festgestellt. Weitere $935(45,0 \%)$ Personen berührten die MNS-Fläche während der Adjustierung über Mund und Nase (BS 2: Adjustierung), 501 (24,1\%) Personen berührten die MNS-Fläche in der Zeit nach der Adjustierung und vor dem Betreten des Lebensmittelgeschäftes (BS 3: Trageverhalten). Insgesamt 116 (5,6\%) Personen zeigten fehlerhaftes Verhalten in jeder der 3 Sequenzen der Beobachtungseinheit. Nahezu die Hälfte $(n=928$; $44,6 \%$ ) aller beobachteten Personen zeigten mindestens ein fehlerhaftes Verhalten innerhalb der Beobachtungseinheit.

Es konnte gefolgert werden, dass das Verhalten bei der Verwendung von MNS gemäß aktuellen Richtlinien stark fehlerbehaftet ist. Offensichtlich besteht für die Entscheidungsträger*innen akuter Handlungsbedarf, und es ist unerlässlich, sich stärker auf zielgruppenspezifische Aufklärungsstrategien hinsichtlich des korrekten Gebrauchs von MNS zu konzentrieren.

\section{Projekt 6}

Das interdisziplinäre Forschungsprojekt Ethische Herausforderungen in der stationären Altenpflege während der Corona-Krise 2020 - Evidenz aus Tirol (EHACK), welches von Juli 2020 bis Juni 2021 durch- 
geführt und vom Land Tirol gefördert wird, verfolgt die Zielsetzung, pflegeethische Herausforderungen für Pflegepersonen in der Langzeitpflege in Tirol während der Corona-Krise zu ermitteln. Dabei wurden im Zeitraum zwischen Oktober 2020 und Februar 2021 einerseits individuelle Erfahrungen, aber auch getroffene Maßnahmen und Lösungsansätze aus der Sicht der Pflegepersonen mittels qualitativer Interviews erhoben. Zudem werden mittels einer OnlineBefragung von Heim- und Pflegedienstleitungen organisationale Aspekte identifiziert, welche pflegeethische Herausforderungen beeinflussen oder bedingen. Die quantitativen Erkenntnisse zielen darauf ab, den organisationalen Rahmen der Interviewergebnisse abzustecken.

Die bereits beendete Interviewstudie schließt 18 problemzentrierte Interviews mit Pflegepersonen sämtlicher Ausbildungsniveaus sowie Sozialbetreuer*innen ein. Der dafür entwickelte Interviewleitfaden fokussiert auf die ethischen Herausforderungen in der Langzeitpflege während der COVID-19-Pandemie wie auch auf die Arbeitsbelastung und das persönliche Erleben der Pandemiesituation seitens der Pflegepersonen.

Erste Analysen dieser Interviewdaten weisen darauf hin, dass Pflegepersonen im Pflegealltag ein moralisches Dilemma wahrnehmen, das sich aus dem Spannungsfeld zwischen dem Respekt vor der Autonomie der Bewohner*innen und dem Schutz vor Ansteckungen der Bewohner*innen ergibt. Die COVID-19Schutzmaßnahmen wie Quarantäne, Testungen und Masken führen teilweise zu herausfordernden Situation mit Bewohner*innen, insbesondere Menschen mit Demenz, was in weiterer Folge Pflegepersonen belastet.

Aus den daraus resultierenden Erkenntnissen sollen Handlungsempfehlungen für zukünftige Krisen, eine Sensibilisierung für pflegeethische Fragestellungen in Pandemiesituation sowie die Erhöhung der Kompetenz im Umgang mit ethischen Problemen und Konflikten im Setting Alten- und Pflegeheime resultieren.

\section{Diskussion}

Die Zusammenfassung der einzelnen Projekte zeigt, dass pflegewissenschaftliche Forschung zu COVID-19 zurzeit in Österreich durchgeführt wird. Die unterschiedlichen Projekte beleuchten die Pflegepraxis im Umgang mit der COVID-19-Pandemie aus verschiedenen Blickwinkeln, wodurch ein umfassender Einblick in die Thematik ermöglicht wird.

Die Ergebnisse des ersten beschriebenen Projekts zeigten deutlich, dass eine höhere Tragedauer der Masken [28] sowie eine Erhöhung des Arbeitsausmaßes [26] signifikant mit einem höheren Stresslevel der Pflegepersonen in Zusammenhang standen. Zusätzlich gaben $31,3 \%$ und 21,7\% der Pflegepersonen an, dass Informationen nicht zeitgerecht und Maßnahmen nicht transparent kommuniziert wurden [29], was wiederum den Stresslevel erhöhen kann. Als Möglichkeit zum Schutz vor psychischer und physischer Überlastung aufgrund von COVID-19 gaben 55\% der Pflegepersonen zusätzliche Pausen und Erholungsphasen zur Stresslinderung an [29], wohingegen psychosoziale Unterstützung oder Sonderurlaube von 34,9\% sowie 33,7\% der Pflegepersonen als Möglichkeit zum Schutz vor Überlastung angegeben wurden [29].

Dies ist mit besonderem Augenmerk zu interpretieren, da die personelle Mindestausstattung in steirischen Pflegeheimen aufgrund von COVID-19 unterschritten werden darf [46]. Im Kontrast dazu, startete Anfang November 2020, vor der zweiten COVID19-Welle, das Bundesministerium eine Kampagne mit dem Titel „Pflege mit Zukunft, Berufe mit Sinn, Vielfalt und Zukunft“ mit Slogans wie „ich arbeite jeden Tag für ein Lächeln mehr“ und „mein Beruf ist meine Leidenschaft“ [47]. Die Diskrepanz zwischen der gelebten Pflegepraxis, sichtbar durch die Ergebnisse der pflegewissenschaftlichen Projekte, und den positiven Suggestionen durch die Medien beim Versuch, den Pflegepersonalnotstand zu verringern, kann hier nur aufgezeigt werden. Dieser Umstand bedarf sehr wohl eines kritischen und gesellschaftlichen Diskurses, insbesondere da zusätzliche Aufgaben und geänderte Verantwortungen in den Bereichen der multiprofessionellen Zusammenarbeit und der Edukation von Patient*innen, wie im Projekt 3 beschrieben, durch die COVID-19-Pandemie an das Pflegepersonal gestellt wurden und werden.

Aufgrund der aktuellen zweiten Welle in Österreich, aber auch im Hinblick auf weitere Wellen oder zukünftige Pandemien sind zukünftig Studien von Interesse, um Vergleiche zu ziehen. Bezogen auf die Langzeiteffekte der Tragedauer von Masken, wären weiterführende Studien sowohl beim Pflegepersonal als auch in der Allgemeinbevölkerung durchaus wünschenswert. Im Hinblick auf den internationalen Pflegepersonalnotstand sollten sich künftige Studien mit effektiven Maßnahmen der Minderung von Stress am Arbeitsplatz von Pflegepersonen beschäftigen.

Ein weiteres Projekt berichtete, dass $45 \%$ der beobachteten Allgemeinbevölkerung zumindest ein fehlerhaftes Verhalten hinsichtlich der Verwendung eines Mund-Nasen-Schutzes vor Lebensmittelgeschäften zeigten. Dies ist insofern von Bedeutung, da auch aktuell von einer fehlerhaften Verwendung der FFP2Masken auszugehen ist. Insbesondere im Hinblick auf bereits weit verbreitete ansteckendere Mutationen des Coronavirus in Österreich ist die adäquate Verwendung von Masken zu forcieren. Internationale Publikationen weisen auf die Wichtigkeit der Gesundheitskompetenz der Allgemeinbevölkerung in der COVID19-Pandemie hin [48-51]. Die „International Health Literacy Association“ kommt zu dem Schluss, dass die epidemiologische Kurve nur dann sinkt, wenn die Allgemeinbevölkerung in der Lage ist, evidenzbasierte Informationen u.a. zur Nutzung von Masken zu 
finden, zu verstehen, zu beurteilen und anzuwenden [50]. Die Ergebnisse der hier beschriebenen Studie geben einen klaren Hinweis darauf, dass in der österreichischen Bevölkerung hinsichtlich der korrekten Nutzung von Masken Verbesserungspotenzial besteht.

Verschiedenste Studien haben bereits gezeigt, wie wichtig Sozialkontakte für kranke alte/ältere und/oder an Demenz erkrankte Personen sind [10]. Eine aktuelle Studie aus Österreich fand heraus, dass zum einen $58 \%$ der älteren Bevölkerung es als belastend empfunden haben, ihre Kinder und Enkelkinder nicht sehen zu können. Gleichzeitig hatten die Personen, die die Maßnahmen als sehr belastend empfunden haben, auch eine geringere Lebenszufriedenheit und mehr depressive Symptome wie Angst und Einsamkeit [52]. In Pflegeheimen wird An-/Zugehörigen eine Schlüsselposition zugeordnet, weil sie in der einzigartigen Situation sind, die emotionalen, sozialen und gesundheitsbezogenen Bedürfnisse der Bewohner*innen verstehen, artikulieren und unterstützen zu können [53].

Das EHACK-Projekt beschäftigt sich mit den ethischen Herausforderungen in der stationären Altenpflege während der COVID-19-Krise 2020. Solche ethischen Herausforderungen wie der Schutz und die Freiheit der Bewohner*innen wurden auch in den veröffentlichten Empfehlungen zu COVID-19-Schutzmaßnahmen für Pflege und Betreuung in teil- und stationären Einrichtungen und mobilen Diensten vonseiten des Bundesministeriums (April 2020) betont. Dort wurde in der Einleitung darauf hingewiesen, dass „die Restriktion von Besuchen oder von gemeinsamen Aktivitäten, das psychische Wohlbefinden der Menschen, insbesondere jener mit demenziellen oder kognitiven Beeinträchtigungen, beeinträchtigen können“ [54]. Die Ergebnisse des EHACK-Projekts könnten in zukünftigen Pandemien Lösungsansätze bieten, um das Wohlbefinden und die Lebensqualität der Bewohner*innen zu steigern.

\section{Schlussfolgerungen}

Dieser Beitrag zielt darauf ab, einen Überblick über pflegewissenschaftliche Projekte und einzelne Studien während der COVID-19-Pandemie zu geben. Damit wird aufgezeigt, dass die Pflegewissenschaft mit ihren Erkenntnissen als Sprachrohr für die Pflegepraxis fungiert und bereit ist, sich in Diskurse einzubringen. Zusätzlich können die Erkenntnisse der präsentierten Projekte als Basis für zukünftige politische Entscheidungen genutzt werden. Zukünftige Projekte hinsichtlich COVID-19 und Pflege, sollten sich evidenzbasierten Handlungsempfehlungen bezüglich der persönlichen Schutzausrüstung (z.B. zu Anwendungsaspekten der Masken, zur Kommunikation mit Masken) und weiteren Aspekten des Arbeitens unter Pandemiebedingungen widmen. Ebenso wären weiterführende Studien in Bezug auf den Zusammenhang des Stresslevels mit dem Berufsausstieg und Maßnahmen, um dem entgegenzuwirken, insbesondere im
Hinblick auf den internationalen Personalnotstand von Interesse. Die Perspektive und die Erfahrungen von An-/Zugehörigen würden den Einblick in die gesundheitliche Versorgung zusätzlich erweitern und sind dementsprechend empfohlen.

Interessenkonflikt M. Hödl, S. Bauer, D. Eglseer, M. Fangmeyer, M. Flatscher-Thöni, J. Kellerer, C. Kreyer, G. Müller, M. Pallauf, M. Rohringer, A. Toromanova und D. Schoberer geben an, dass kein Interessenkonflikt besteht.

\section{Literatur}

1. Hughes R. Patient safety and quality: an evidence-based handbookfor nurses. AHRQPublication, Bd.08-0043. Rockville: Agency for Healthcare Research and Quality; 2008.

2. Al Jazeera. Coronavirus: Worldwide applause for frontline medical staff, 30. March 2020, A.J.M. Network, Editor. 2020. https://www.aljazeera.com/programmes/ newsfeed/2020/03/coronavirus-worldwide-applause frontline-medical-staff-200330111116862.html. Zugegriffen: 10.09.2020.

3. WHO. The world health report 2006: working together for health. Geneva:World Health Organization; 2006.

4. WHO. The global strategic directions for strengthening nursing and midwifery:2016-2020. Geneva:WHO;2016.

5. Petzold MB, Plag J, Ströhle A. Dealing with psychological distress by healthcare professionals during the COVID-19 pandemia. Nervenarzt. 2020;91(5):417-21.

6. Inter-Agency Standing Committee. Addressing mental health and psychosocial aspects of COVID-19 outbreak. 2020. https://interagencystandingcommittee.org/ iasc-reference-group-mental-health-and-psychosocialsupport-emergency-settings/interim-briefing. Zugegriffen: 10.06.2021.

7. Lai J, et al. Factors associated with mental health outcomes amonghealth care workers exposed to Coronavirus disease. JAMANetw Open. 2019;2020(3):3-e203976.

8. Mo Y, et al. Work stress among Chinese nurses to support Wuhan for fighting against the COVID-19 epidemic. J Nurs Manag. 2020;28(5):1002-9.

9. Deutsches Netzwerk Evidenz-basierte Medizin. COVID-19: Wo ist die Evidenz? 2020.

10. Schrems BM. Nach der Krise ist vor der Krise oder: Nothing changes if nothing changes. Pflege. 2021;34(1):1-2.

11. Hoedl M. Die COVID-19 Krise als Chance für die Pflegepraxis und -wissenschaft: eine Reflexion. Pflegenetz. 2021;01:19-21.

12. Bundesministerin für Arbeit, G.u.S.. Coronavirus-Taskforce. 2021.

13. Donabedian, A. The quality of care: How can it be assessed? JAMA. 1988;260(12):1743-8.

14. WHO. Infection prevention and control during health care when COVID-19 is suspected: interim guidance, 19 March 2020. 2020. https://www.who.int/publications/i/ item/10665-331495.Zugegriffen: 10.06.2021.

15. WHO. Advice on the use of masks in the context of COVID19: interim guidance, 6 April 2020. Geneva: World Health Organization; 2020.

16. Bundesministerium für Soziales Gesundheit Pflege und Konsumentenschutz. Empfehlung zu COVID-19 Schutzmaßnahmen für Pflege und Betreuung [in German]. 2020. Republic Austria:https://www.sozialministerium.at/ Informationen-zum-Coronavirus/Coronavirus----Fach informationen.html.Zugegriffen: 10.06.2021. 
17. Bundesministerium für Soziales Gesundheit Pflege und Konsumentenschutz. Übersicht Einsatzbereiche verschiedener Maskenarten und Mund-Nasen-Schutzes im Gesundheits-/Sozialbereich. H. Federal Ministry for Social Affairs, Care and Consumer Protection, Editor. 2020, RepublicAustria:Vienna.

18. Bundesministerium für Soziales Gesundheit Pflege und Konsumentenschutz. Empfehlung zu COVID-19 Schutzmaßnahmen für Krankenanstalten. H. Federal Ministry for Social Affairs, Care and Consumer Protection, Editor. 2020, Republic Austria:Vienna.

19. Bundesministerium für Soziales Gesundheit Pflege und Konsumentenschutz. Handlungsempfehlung: Informationen für Personenbetreuungskräfte in der 24-Stunden-Betreuung. H. Federal Ministry for Social Affairs, Care and Consumer Protection, Editor. 2020, Republic Austria: Vienna.

20. Ong J, et al. Headaches associated with personal protective equipment-A cross-sectional study among frontline healthcare workers during COVID-19. Headache. 2020;60(5):864-77.

21. Klein EM, et al. The German version of the Perceived Stress Scale-Psychometric characteristics in a representative German community sample. BMC Psychiatry. 2016;16:159.

22. Aalaa M, et al. Influence of MTHFR gene variations on perceived stress modification: Preliminary results of NURSE study. Med J Islam Repub Iran. 2017;31:128.

23. Cicolini G, et al. Validation of the Italian version of the Stanford Presenteeism Scale in nurses. J Nurs Manag. 2016;24(5):598-604.

24. Montanari KM, et al. Mindfulness: assessing the feasibility of a pilot intervention to reduce stress and burnout. J Holist Nurs. 2019;37(2):175-88.

25. IBM Corp. IBM SPSS Statistics for Windows Version 26.0. New York: IBMCorp; 2019.

26. Hoedl M, Bauer S, Eglseer D. Influence of nursing staff working hours on the stress level during the COVID-19 pandemic: a cross-sectional online survey. 2020. https:// doi.org/10.1101/2020.08.12.20173385.

27. Hoedl M, Eglseer D, Bauer S. COVID-19 among nursing staff: settings and regional differences. 2020. https:// doi.org/10. 1101/2020.08.14.20174797.

28. Hoedl M, Eglseer D, Bauer S. Associations between personal protective equipment and nursing staff stress during the COVID-19 pandemic. 2020. https://doi.org/10.1101/2020. 08.06.20164129.

29. Bauer S, Eglseer D, Hoedl M. Pflege während der COVID19 Pandemie - Eine besondere Herausforderung. ProCare. 2020;25:48-53.

30. Bundesministerium für Soziales Gesundheit Pflege und Konsumentenschutz. Pflegepersonal-Bedarfsprognose für Österreich. 2019. https://broschuerenservice. sozialministerium.at/Home/Download?publicationId $=722$. Zugegriffen: 10.06 .2021 .

31. Statistics Austria. Nichtärztliches Gesundheitspersonal 2018 in Krankenanstalten nach Geschlecht, Fachrichtung und Bundesland.2018in.

32. Koehler S, Meyer A. Psychische Belastung und Beanspruchung. Hamburg: Berufsgenossenschaft für Gesundheitsdienstund Wohlfahrtspflege (BGW);2017.

33. Mayring P. Qualitative Inhaltsanalyse. In: Mey MKG, Hrsg. Handbuch Qualitative Forschung in der Psychologie. Wiesbaden:VS;2010.

34. Schreier M. Qualitative content analysis in practice. Thousand Oaks: SAGE; 2012.

35. Hussy W, Schreier M, EchterhoffG. Qualitative Forschungsmethoden. In: Forschungsmethoden in Psychologie und
Sozialwissenschaften für Bachelor. Berlin, Heidelberg, New York: Springer; 2010.

36 . Neuendorf KA. The content analysis guidebook. Thousand Oaks:SAGE; 2016.

37. Hoedl M. Mehr als Applaus, in AirCampus, L. März, Editor. 2020. https://www.aircampus-graz.at/podcasts/pflege/. Zugegriffen: 10.06.2021.

38. RossacherT.Sobelastendist Corona für diePfleger, in Kleine Zeitung. 2020. https://www.kleinezeitung.at/steiermark/ 5873763/Grosse-CoronaUmfrage_Das-macht-demPflegePersonal-zu-schaffen.Zugegriffen: 01.10.2020.

39. Centers for Disease Control and Prevention. Stephen B. Thacker CDC Library. 2020. https://www.cdc.gov/library/ researchguides/2019novelcoronavirus/researcharticles. html.Zugegriffen: 10.06.2021.

40. The Cochrane Collaboration. Cochrane COVID-19-Study register.2021.https:// covid-19.cochrane.org/?pn=1.Zugegriffen: 10.06.2021.

41. WHO. WHO-COVID-19-Global literature on coronavirus disease. 2021. https://search.bvsalud.org/globalliterature-on-novel-coronavirus-2019-ncov/. Zugegriffen: 10.06.2021.

42. COVID-NMA. COVID-NMA initiative: Covid-19 open living evidence synthesis to inform decision. 2021. https:/ / covidnma.com/.Zugegriffen: 10.06.2021.

43. Cochrane Collaboration. Guidance for the production and publication of Cochrane living systematic reviews: Cochrane reviews in living mode. 2019. https:// community.cochrane.org/sites/default/files/uploads/ inline-files/Transform/201912_LSR_Revised_Guidance. pdf.Zugegriffen: 10.06.2021.

44. Seneviratne CJ, et al. Efficacy of commercial mouth-rinses on SARS-CoV-2 viral load in saliva: randomized control trial in Singapore. Infection. 2020;49(2):305-11.

45. Kellerer JD, Rohringer M, Deufert D. Behavior in the use of face masks in the context of COVID-19. Public Health Nurs. 2021.https://doi.org/10.1111/phn.12918.

46. Steiermärkische Landesregierung. Verordnung der Steiermärkischen Landesregierung vom 23. November 2017 über die Personalausstattung in Pflegeheimen. In: Personalausstattungsverordnung 2017 -PAVO. LGBl, Bd.99/2017. Graz: SteiermärkischeLandesregierung; 2020.

47. Bundesministerium für Soziales Gesundheit Pflege und Konsumentenschutz. Pflege - Berufe mit Sinn, Vielfalt und Zukunft. 2020. https://www.sozialministerium.at/ Themen/Pflege/Pflege-mit-Zukunft.html. Zugegriffen: 10.06.2021.

48. Lazcano-Ponce E, Alpuche-Aranda C. Public health literacy in the face of the Covid-19 pandemic emergency. Salud Publica Mex. 2020;62(3):331-40.

49. Sørensen K, et al. Rebranding social distancing to physical distancing: calling for a change in the health promotion vocabulary to enhance clear communication during a pandemic. Glob Health Promot. 2021;28(1):5-14.

50. International Health Literacy Association. IHLA Statement to the WHO on health literacy as an essential lifesaving strategy during the pandemic. 2020. http://www. ihlasummit2021.org/downloads/IHLA\%20Statement\%20 to\%20WHO\%20on\%20health\%20literacy.pdf.Zugegriffen: 10.06.2021.

51. Haischer MH, et al. Who is wearing a mask? Gender-, age-, and location-related differences during the COVID-19 pandemic. PLoS One. 2020;15(10):e240785.

52. Stolz R. Coronakrise: Einschränkungen für Ältere Belastend. 2020. Science.ORF.at. https://science.orf.at/stories/ 3201181/.Zugegriffen: 10.06.2021. 
53. Reinhard S, et al. Supporting family caregivers in providing care. In: Patient safety and quality: an evidence-based handbook for nurses. Rockville: Agency for Healthcare Research and Quality (US);2008.

54. Federal Ministry for Social Affairs, H., Care and Consumer Protection. Empfehlung zu COVID-19 Schutzmaßnahmen für Pflege und Betreuung. 2020. https://www. sozialministerium.at/Informationen-zum-Coronavirus/
Coronavirus---Fachinformationen.html, Republic Austria.Zugegriffen: 10.06.2021.

Hinweis des Verlags Der Verlag bleibt in Hinblick auf geografische Zuordnungen und Gebietsbezeichnungen in veröffentlichten Karten und Institutsadressen neutral. 